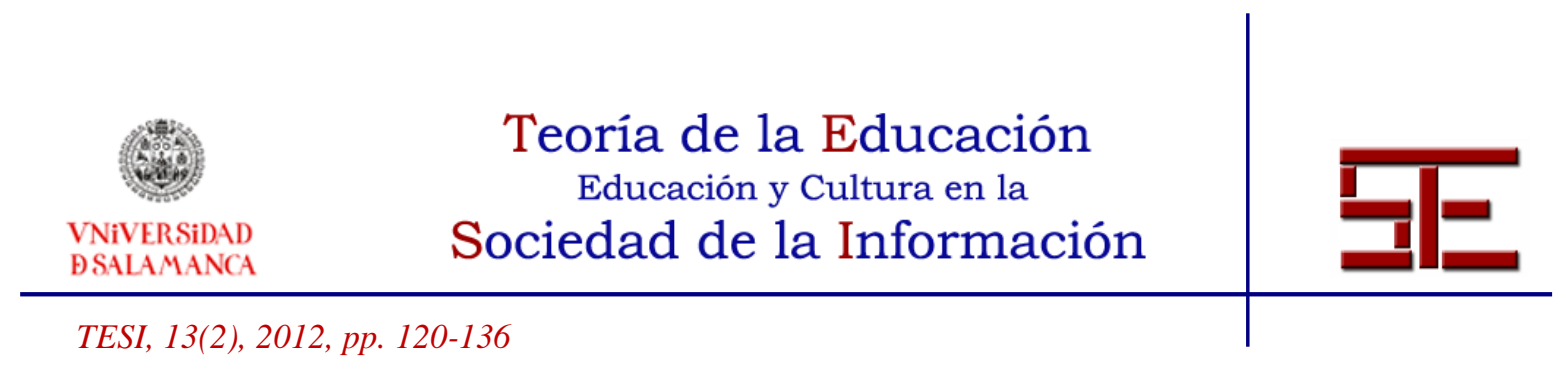

\title{
LA ROBÓTICA COMO UN RECURSO PARA FACILITAR EL APRENDIZAJE Y DESARROLLO DE COMPETENCIAS GENERALES
}

Resumen: La creciente importancia que tiene la tecnología en el mundo hoy en día y su continuo desarrollo, hace que la tecnología, en sí misma, se convierte en parte integral del proceso de formación en la niñez y la juventud. Por esta razón es importante desarrollar propuestas en las que se ofrezca a niños y jóvenes la posibilidad de entrar en contacto con las nuevas tecnologías; esto es posible a través del manejo de herramientas de software y hardware, como prototipos robóticos y programas especializados con fines pedagógicos.

Este artículo muestra la importancia que tiene el uso de la robótica como una herramienta de aprendizaje y presenta las etapas típicas que se deben afrontar al implementar proyectos de robótica educativa en el aula de clase. También se da a conocer un proyecto de robótica educativa denominado "Mundo Robótica" el cual busca involucrar la robótica en el aula de clase por medio de actividades prácticas y recursos de aprendizaje articulados desde una plataforma virtual.

Palabras clave: robótica educativa; ambientes de aprendizaje.

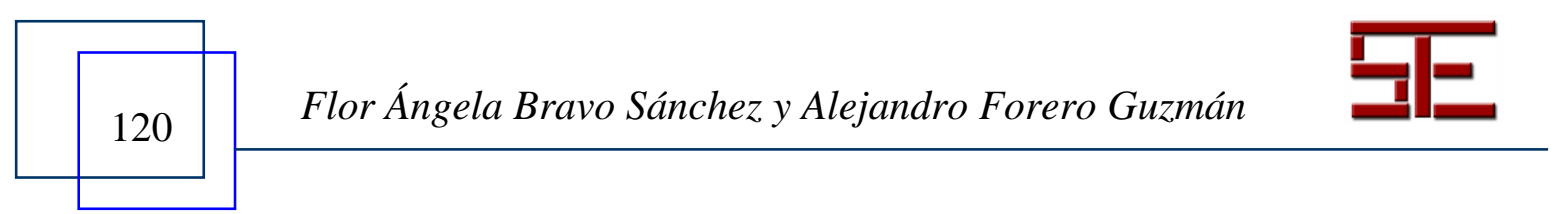




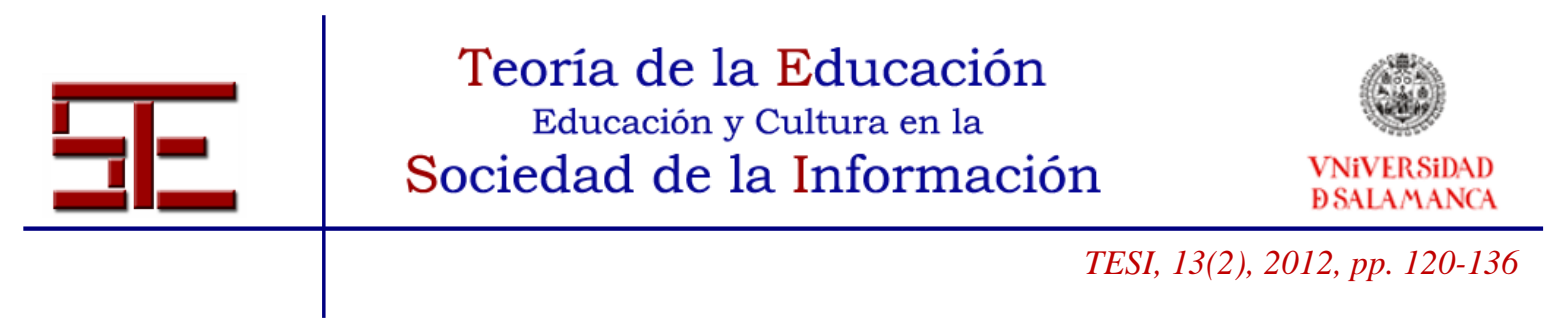

\title{
ROBOTICS AS A RESOURCE TO FACILITATE THE LEARNING AND GENERAL SKILLS DEVELOPMENT
}

\begin{abstract}
The growing importance of technology in the world today and its continuous development, makes the technology becomes an integral part of the formation process in childhood and youth.

For this reason is important to develop proposals that are offered to children and young people to come into contact with new technologies, that is possible through the use of software and hardware tools, such as robotic prototypes and specialized programs for educational purposes.

This paper shows the importance of the use of robotics as a learning tool and presents the typical stages that must be confronted in implementing educational robotics projects in the classroom. It also presents an educational robotics project called "Mundo Robótica" which seeks to involve robotics in the classroom through practical activities and learning resources, all this is articulated from a virtual platform.
\end{abstract}

Keywords: robotics education; learning environment

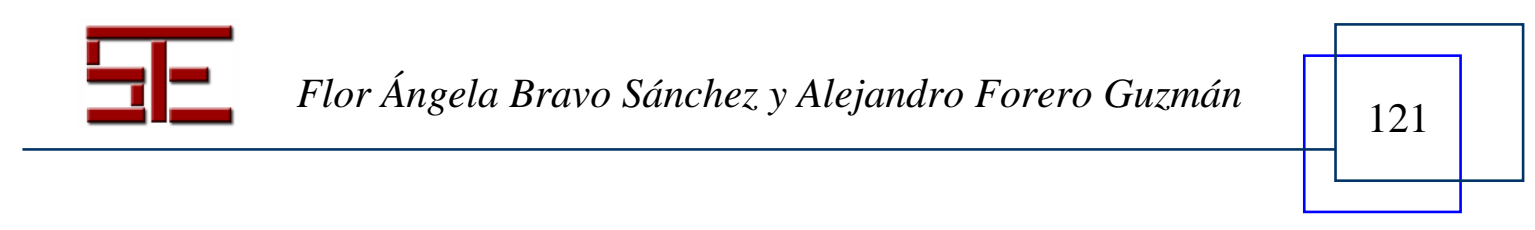




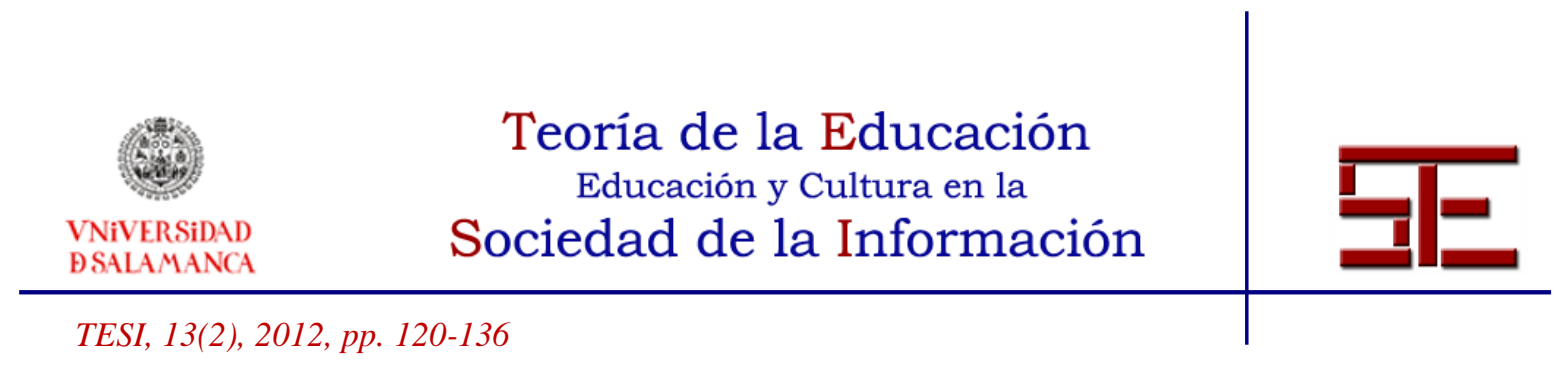

\section{LA ROBÓTICA COMO UN RECURSO PARA FACILITAR EL APRENDIZAJE Y DESARROLLO DE COMPETENCIAS GENERALES}

Fecha de recepción: 1/05/2012; fecha de aceptación: 03/07/2012; fecha de publicación: 26/07/2012

Flor Ángela Bravo Sánchez

bravof@javeriana.edu.co

Pontificia Universidad Javeriana. Bogotá, Colombia.

Alejandro Forero Guzmán

alejandro.forero@javeriana.edu.co

Pontificia Universidad Javeriana. Bogotá, Colombia.

\section{1.- INTRODUCCIÓN}

La robótica en el ámbito educativo se convierte en un recurso para facilitar el aprendizaje y desarrollar competencias generales como la socialización, la creatividad y la iniciativa, que permitan al estudiante dar una respuesta eficiente a los entornos cambiantes del mundo actual. La presencia de la robótica en el aula de clase no intenta formar a los estudiantes en la disciplina de la robótica propiamente dicha, sino aprovechar su carácter multidisciplinar para generar ambientes de aprendizaje donde el estudiante pueda percibir los problemas del mundo real, imaginar y formular las posibles soluciones y poner en marcha sus ideas, mientras se siente motivado por temas que se van desarrollando (Del Mar, 2006; Aliane, 2007).

Los ambientes de aprendizaje permiten activar procesos cognitivos y sociales que propician un aprendizaje significativo en el estudiante y las destrezas necesarias para desempeñarse adecuadamente en el contexto diverso y complejo que requiere la sociedad. Estos espacios son generados gracias a las relaciones e interacciones que ocurren en el aula de clase entre los estudiantes y docentes, y entre ellos con los recursos con los que se cuenta (Acuña, 2006). El principal objetivo de los ambientes de aprendizaje es convertir el aula de clases en un laboratorio de exploración y experimentación en donde los estudiantes se pregunten constantemente el cómo y el por qué de las cosas en su entorno; en particular, se quiere que las nuevas generaciones se cuestionen respecto a los diferentes elementos que podemos encontrar en el entorno tecnológico actual, pero, sin una excusa como la robótica, estos elementos suelen pasar desapercibidos. Así pues, la robótica educativa pretende despertar en ellos el interés por los temas de clase y facilitar la comprensión de una variedad de conceptos y fenómenos.

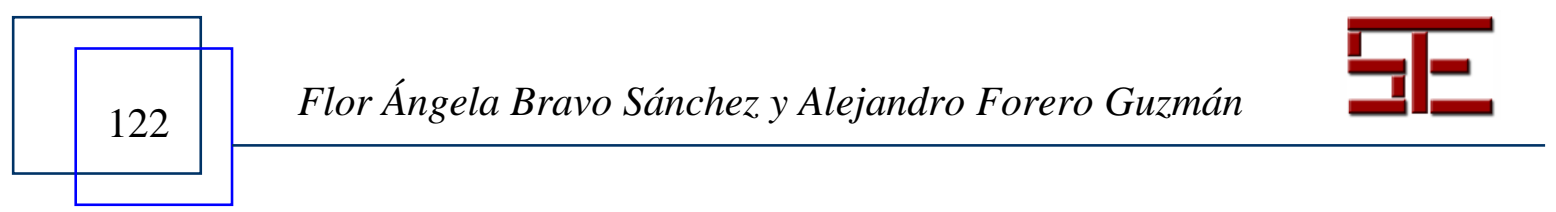




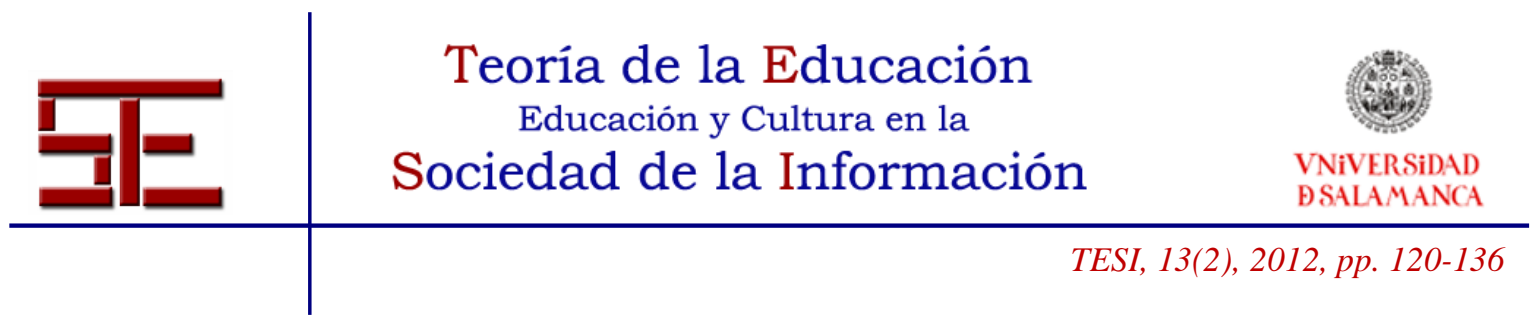

La puesta en marcha de un proyecto de robótica involucra diversas áreas del conocimiento, tales como las matemáticas, la física, la electrónica, la mecánica y la informática, eso sin contar el área propia de la aplicación (p.e j. la cadena de suministros, en una empresa logística que almacena y reparte mercancías; o la medicina, en una aplicación médica). Este confluir de muchas disciplinas la convierte en una gran alternativa integradora para la enseñanza. Pero la robótica no solo tiene la ventaja de integrar múltiples áreas del conocimiento, quizá su mayor cualidad en el ambiente educativo sea lo atractiva que resulta para jóvenes y adultos. Evidencia de este atractivo es la multiplicidad de películas cuya trama gira, de una u otra forma, alrededor de los robots. Todo esto lleva a que la presencia de la robótica en el aula de clase ofrezca a niños, niñas y jóvenes la posibilidad de interactuar con este elemento motivador, que además de centrar el interés de quien aprende en los temas que se enseñan, conecta a los estudiantes con las nuevas tecnologías mientras se le imprime sentido a los saberes que se pretenden enseñar.

En este artículo se exploran los beneficios que trae la aplicación de la robótica en procesos de aprendizaje y se presentan las etapas típicas que se deben afrontar para generar ambientes de aprendizaje interdisciplinarios basados en la robótica. Adicionalmente se presenta un proyecto de robótica educativa denominado Mundo Robótica, que articula desde una plataforma virtual diferentes recursos de aprendizaje basados en robótica.

El resto del presente artículo se divide así: en la sección 2 se presenta una breve introducción a la robótica educativa. En la sección 3 se exploran algunas de las teorías pedagógicas vinculadas a la robótica educativa. En la sección 4 se presentan las etapas que hay que afrontar para poder generar ambientes de aprendizaje interdisciplinarios basados en la robótica. En la sección 5 se presenta el proyecto Mundo Robótica. Finalmente en la sección 6 se presentan las conclusiones y trabajos futuros que podrían desprenderse desde la implementación del proyecto Mundo Robótica.

\section{2.- LA ROBÓTICA EDUCATIVA}

La robótica educativa también conocida como robótica pedagógica es una disciplina que tiene por objeto la concepción, creación y puesta en funcionamiento de prototipos robóticos y programas especializados con fines pedagógicos (Ruiz-Velasco, 2007). La robótica educativa crea las mejores condiciones de apropiación de conocimiento que permite a los estudiantes fabricar sus propias representaciones de los fenómenos del

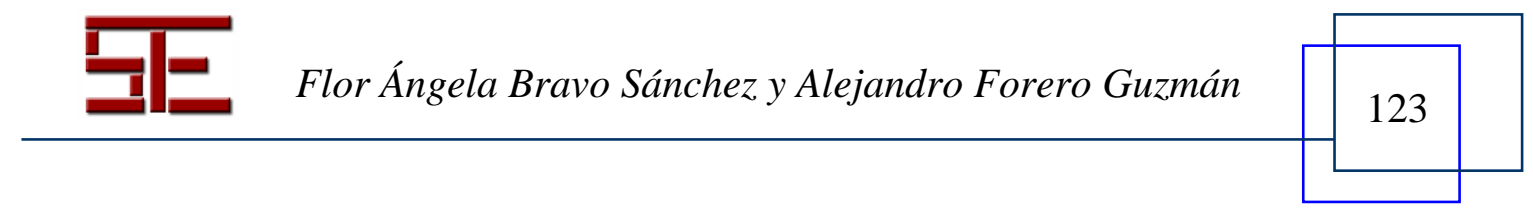




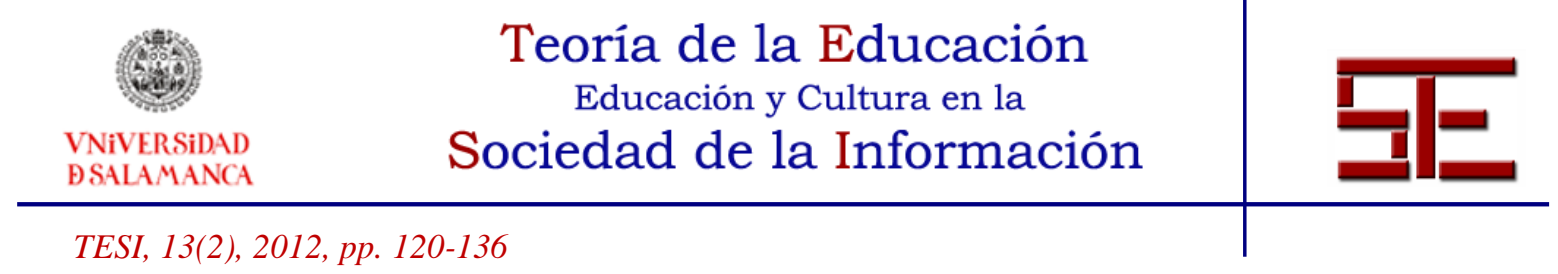

mundo que los rodea, facilitando la adquisición de conocimientos acerca de estos fenómenos y su transferencia a diferentes áreas del conocimiento.

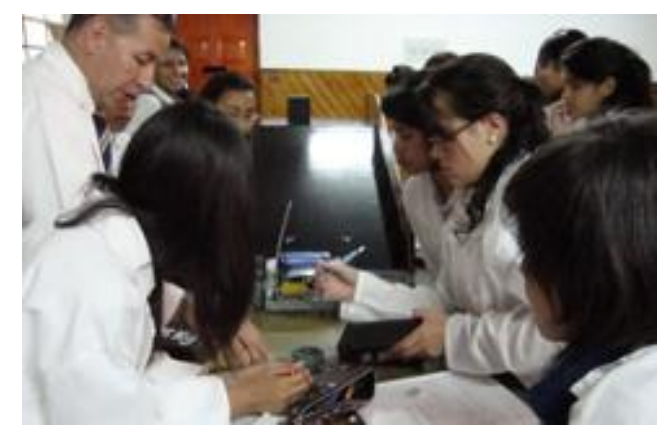

En la imagen se observa a estudiantes del colegio Sagrados Corazones de Mosquera desarrollando una actividad práctica con robótica para su clase de física.
A través de la robótica educativa el docente puede desarrollar de forma práctica y didáctica aquellos conceptos teóricos que suelen ser abstractos y confusos para los estudiantes; usar está estrategia tiene la ventaja adicional de simultáneamente despertar el interés del estudiante por esos temas, al tiempo que pone de manifiesto la relación entre el contexto tecnológico en el que se desenvuelve la vida actual y los temas que se enseñan. En este sentido, un ambiente de aprendizaje con robótica educativa, es una experiencia que contribuye al desarrollo de nuevas habilidades, nuevos conceptos, fortalece el pensamiento sistémico, lógico, estructurado y formal del estudiante, al tiempo que desarrolla su capacidad de resolver problemas concretos, dando así una respuesta eficiente a los entornos cambiantes del mundo actual (Odorico, 2004).

Una característica especial que tiene la robótica educativa es la capacidad de mantener la atención del estudiante. El hecho de que el estudiante pueda manipular y experimentar con estas herramientas de aprendizaje basadas en robótica hace que pueda centrar sus percepciones y observaciones en la actividad que está realizando. Un testimonio en este sentido lo dan Pierre Nonnon y Jean Pierre Theil, quienes afirman que el uso de herramientas robóticas favorece el proceso de enseñanza-aprendizaje, pues permite fácilmente la integración de lo teórico con lo práctico, el desarrollo de un pensamiento sistémico y la adquisición de nociones científicas (Ruiz-Velasco, 2007).

Para poder aplicar proyectos de robótica en el aula de clase es necesario disponer de diferentes herramientas de software y/o hardware que permitan al estudiante construir o simular diferentes prototipos robóticos. Los kits comerciales de robótica son una gran opción para involucrar la robótica en el aula de clase. Estas herramientas educativas permiten a personas de todas las edades construir diferentes prototipos robóticos sin necesidad de tener conocimientos avanzados en mecánica, electrónica o programación. Actualmente el mercado ofrece una variedad de herramientas para el aprendizaje y la

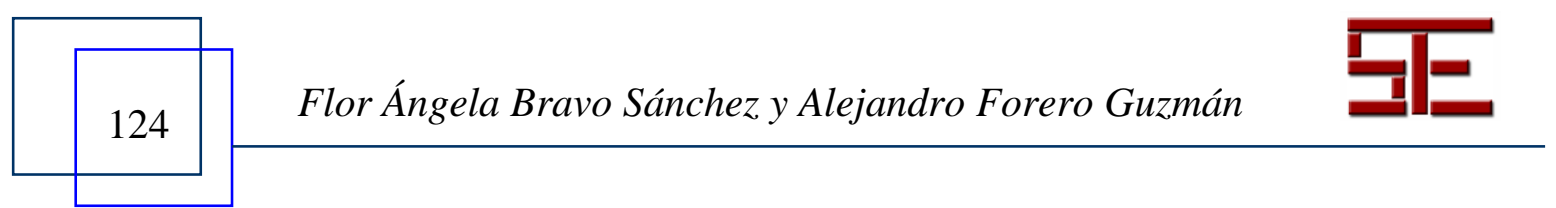




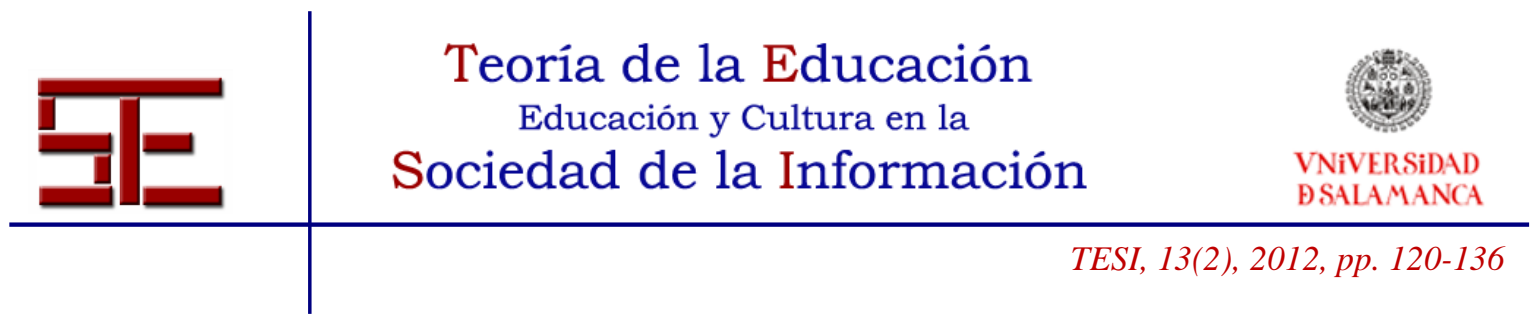

estimulación. Entre los kits más conocidos en el mercado se pueden comentar: los Bee-Bot, Parallax Scribbler, VEX Robotics, Fishertechnik PROFI y Computing, LEGO MINDSTORMS education, LEGO WeDo, LEGO NXT, sistema BIOLOID, DYNAMIXEL y DARwIn-OP.

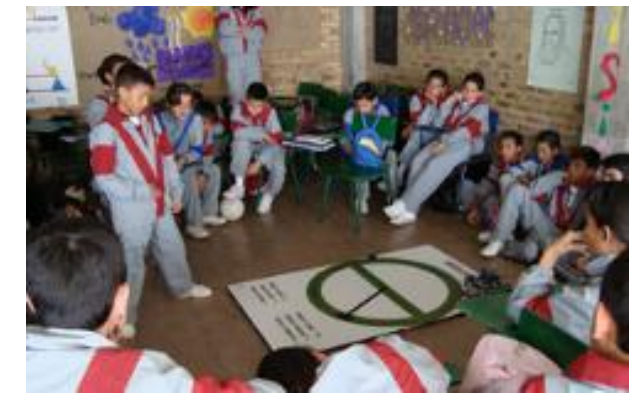

En el mercado también se pueden encontrar programas especializados

En la imagen se observa a estudiantes del colegio Parroquial Santiago Apóstol de Funza desarrollando una actividad práctica con robótica para su clase de Matemáticas.

en robótica que permiten a niños y jóvenes controlar y simular diferentes prototipos robóticos. Se pueden destacar los siguientes programas educacionales: NXT-G Educación, ROBOTC, ROBOLAB, Microsoft Robotics Developer Studio, Scratch, RoboRealm, LabVIEW y MATLAB (Lrobotikas, 2011).

Aunque no todos los colegios están en la capacidad de adquirir uno o más kits de robótica, esto no debe ser un impedimento para que los estudiantes entren en contacto con estas tecnologías y en particular está metodología de integrar la práctica con la teoría de que se aprende. Se puede hacer uso del material reciclado de diferentes dispositivos electrónicos para la construcción de los prototipos robóticos, o alterar los juguetes tecnológicos para construir los prototipos de los robots (Heli: Reciclaje y proyectos electrónicos, 2012). En Colombia, el programa Computadores Para Educar desarrolla plataformas de Robótica y Automática Educativa, como una estrategia para el aprovechamiento de los residuos electrónicos. Este programa recupera un gran número de partes eléctricas, mecánicas y componentes electrónicos que se utilizan para la construcción de kits de robótica educativa y plataformas de desarrollo. El objetivo de estas herramientas de robótica de material reciclado es la generación de ambientes de aprendizaje en las escuelas públicas beneficiarias del programa en todo Colombia (Computadores para Educar, 2008).

A nivel de proyectos con Hardware Libre, se destacan los desarrollos basados en el sistema Arduino (Arduino, 2006), que es una plataforma de desarrollo abierta que facilita el uso de la electrónica en proyectos multidisciplinares. Lo atractivo del sistema Arduino es que permite programar y controlar prototipos robóticos sin necesidad de tener conocimientos avanzados en electrónica o programación. Los sistemas Arduinos

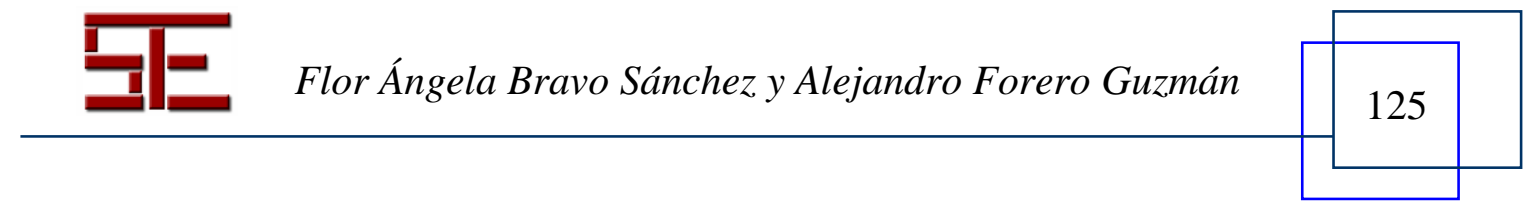




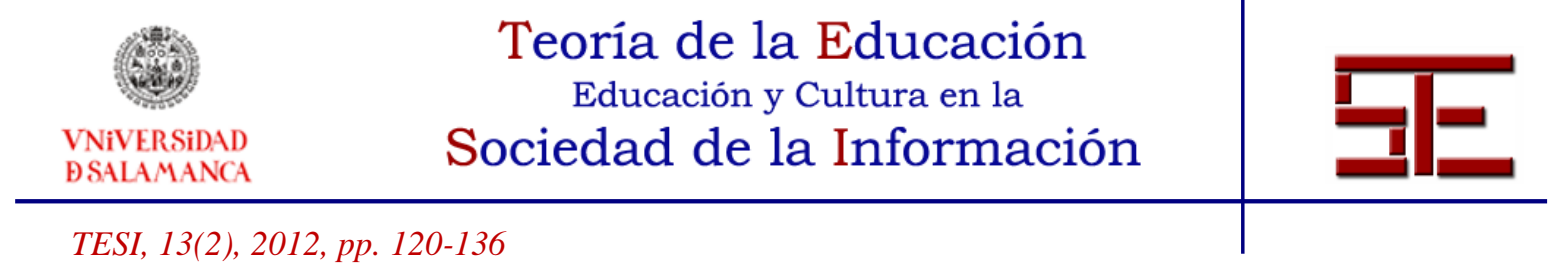

pueden ser programados en cualquier Sistema Operativo (Windows, Mac y Linux) en un entorno propio en lenguaje C/C++ o en Scratch for Arduino-S4A para niños (Ruiz, 2012). Una de las grandes fortalezas de los sistemas Arduino es su capacidad de integración con sistemas mecánicos de LEGO Education, LEGO Technic y Fischertechnik, con sensores y actuadores de Hitec Robotics o Mindsensors, con los motores y baterías de LEGO Power Functions y con dispositivos Android.

En herramientas software libre, se destaca OpenQbo Robotic, una distribución de Linux basada en Ubuntu, que incluye software y aplicaciones dirigidas a la robótica. Algunas aplicaciones predeterminadas en OpenQbo Robotic son: Gnome (entorno gráfico), Festival (motor de síntesis de habla), Julius (motor de reconocimiento vocal), OpenCV (sistema de visión artificial), ROS (sistema meta-operativo), SSH (intérprete de órdenes bajo protocolo seguro), Apache (servidor Web), Mysql Server (servidor de base de datos) y Qbo GUI (interfaz gráfica entre el robot y el usuario) (OPENQBO, 2010).

\section{3.- TEORÍAS PEDAGÓgICAS VINCULAdAS A LA ROBÓtICA EDUCATIVA}

La robótica educativa está fuertemente vinculada con las teorías del constructivismo y la pedagogía activa. La teoría constructivista de Jean Piaget (1976) asegura que el aprendizaje no es resultado de una transferencia de conocimiento sino que es un proceso activo de construcción del aprendizaje basado en experiencias (Acuña, 2004). El constructivismo sostiene que el aprendizaje se manifiesta a medida que el estudiante interactúa con su realidad y realiza concretamente actividades sobre ella. Desde el punto de vista de la teoría constructivista, el uso de herramientas tecnológicas en el aula de clase aporta una manera alternativa de aprender y crea en los estudiantes experiencias para la construcción de conocimientos (Hernández, 2008).

Los ambientes de aprendizaje generados por la robótica educativa están basados fundamentalmente en la acción de los estudiantes. Los proyectos de robótica educativa posicionan al estudiante en un rol activo y protagónico en su propio proceso de aprendizaje pues permiten al estudiante pensar, imaginar, decidir, planificar, anticipar, investigar, hacer conexiones con el entorno, inventar, documentar y realimentar a otros compañeros; en la vivencia de todo este proceso, desarrollarán diversos conocimientos y habilidades esenciales para desenvolverse eficientemente ante los retos y desafíos que impone el mundo actual (Acuña, 2004).

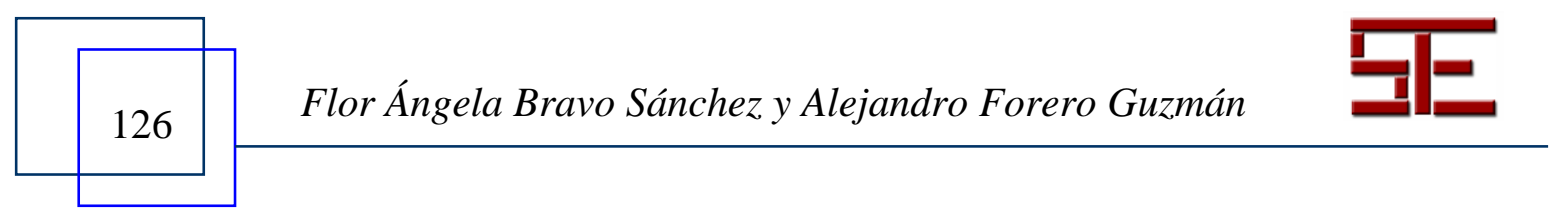




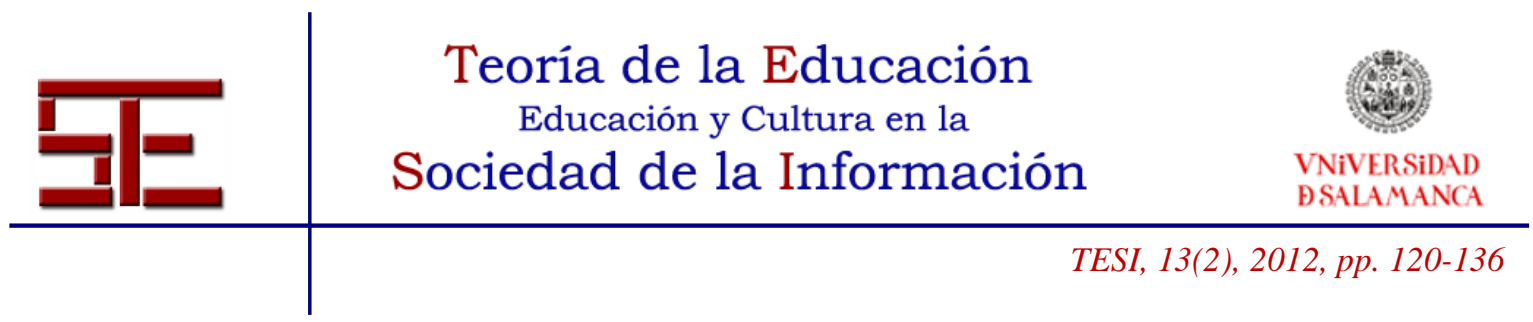

\section{4.- IMPLEMENTACIÓN DE PROYECTOS DE ROBÓTICA EDUCATIVA EN EL AULA DE CLASE}

El proceso necesario para la implementación de proyectos de robótica educativa en el aula de clase puede dividirse en cuatro etapas; el punto de partida depende del grado de concientización que tenga la institución educativa que pretende implementar estos ambientes de aprendizaje o las experiencias previas que se hayan realizado en la institución. A continuación se detalla cada una de las cuatro etapas:

\section{1.- Etapa de integración de recursos tecnológicos basados en robótica al currículo}

En esta etapa se sitúan muchos colegios donde se emplea algún tipo de herramientas de robótica, estas no suelen aprovecharse al máximo. El principal inconveniente que se presenta al momento de involucrar la robótica en la educación es que se considera como una actividad extracurricular; los colegios crean clubes o talleres de robótica para un grupo limitado de estudiantes o intentan involucrar la robótica a través de algunos proyectos en clase de tecnología, pero sin articularla con las demás áreas del conocimiento, desaprovechando así las posibilidades integradoras y motivacionales que trae el uso de esta tecnología en el aula de clase.

Es importante superar el paradigma que se tiene de la robótica como una actividad extracurricular y reconocer la robótica como una herramienta de aprendizaje, que permite generar interesantes ambientes interdisciplinarios de aprendizaje donde el estudiante como actor principal de su aprendizaje, pueda crear sus propias ideas de los conceptos que están siendo impartidos, al tiempo que los relaciona con su propia realidad.

Hay que tomar conciencia de las ventajas y beneficios que se tienen cuando se hace uso de esta tecnología en el aula de clase como un componente más del proceso de aprendizaje. Se debe evaluar el plan curricular e involucrar proyectos basados en robótica en las diferentes asignaturas y fortalecer así el proceso de enseñanzaaprendizaje del estudiante.

\section{2.- Etapa de restructuración en las prácticas pedagógicas}

Aplicar la robótica en el aula de clase requiere un cambio en las prácticas pedagógicas. Se debe dejar a un lado el esquema tradicional del aula de clase, donde el papel y el lápiz tienen el protagonismo principal y establecer una nueva metodología de

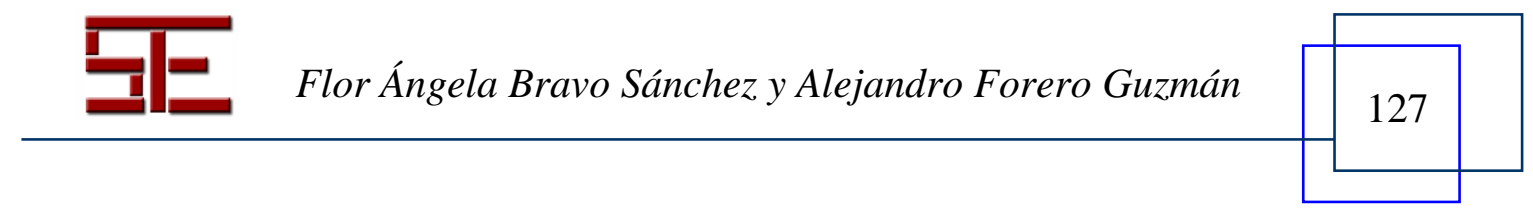




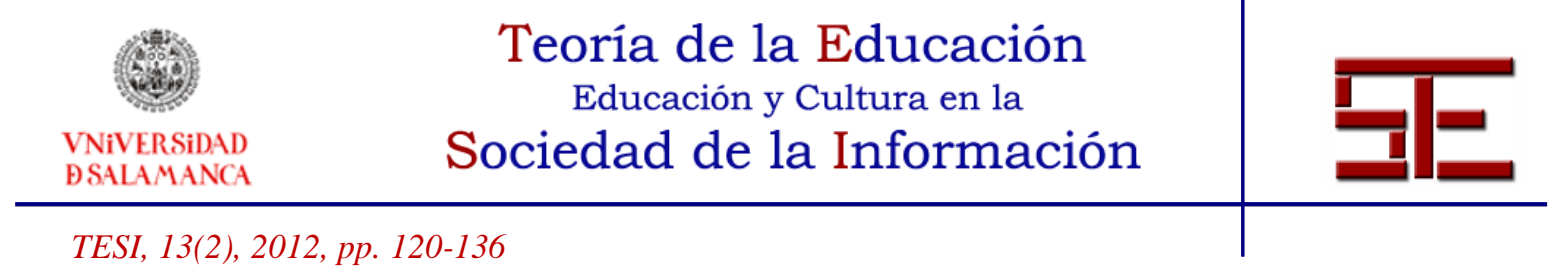

aprendizaje que fortalezca los procesos de enseñanza-aprendizaje del estudiante a través del uso de prototipos robóticos y programas especializados con fines pedagógicos.

Este nuevo ambiente tecnológico requiere un cambio de actitud tanto del estudiante como del docente. En esta etapa, los estudiantes deben adoptar un rol más activo y ser protagonistas de su propio proceso de aprendizaje, mientras que el docente debe asumir el rol de mediador del aprendizaje, incentivando a los alumnos a la búsqueda del conocimiento. Se deben fomentar el uso de herramientas de búsqueda y participación en Internet para enriquecer la experiencia y alcanzar una visión integradora que no se limita a las actividades dentro del aula. La mediación de los docentes tiene la intención de organizar los contextos y orientar los procesos de aprendizaje que permitan una comprensión profunda de temas tratados (Acuña, 2004). Sin embargo es importante resaltar el protagonismo del estudiante, pues contrario a modelos anteriores, las experiencias propuestas no se deben agotar en la organización del docente, ese debe ser solo el elemento inicial, motivador, que despierta la curiosidad de los estudiantes, quienes actuando como grupo enriquecen la actividad.

\section{3.- Etapa de instrumentación}

Para llevar la robótica al aula de clase es necesario disponer de diferentes herramientas de software y hardware que permitan la construcción y programación de diferentes prototipos robóticos. Hay que tener en cuenta que se debe disponer de más de una herramienta robótica para trabajar con un grupo numeroso de estudiantes. Cuando se dispone de una sola herramienta, es muy difícil captar la atención de todos los estudiantes, que pueden estar muy motivados al iniciar la actividad, pero, al perder la oportunidad de actuar directamente, pierden interés cuando no pueden apreciar claramente las actividades realizadas por el prototipo robótico. Igual sucede cuando la participación en la actividad se ve reducida a unos pocos estudiantes.

Uno de los inconvenientes que se presenta al trabajar con herramientas de robótica es que se cree que solo deben ser manipuladas por personas que tengan experiencia y conocimientos de robótica y programación. La falta de conocimiento y experiencia en el manejo de estas herramientas robóticas por parte de los docentes puede llegar a generar en ellos temor e inseguridad al momento de usarlas frente a sus estudiantes. Por eso, en esta etapa es importante realizar diferentes actividades con los docentes para capacitarlos en el manejo de estas herramientas para concientizarlos de sus bondades y dejar en claro que no se requiere ser experto para su manipulación. Todo esto debe darse

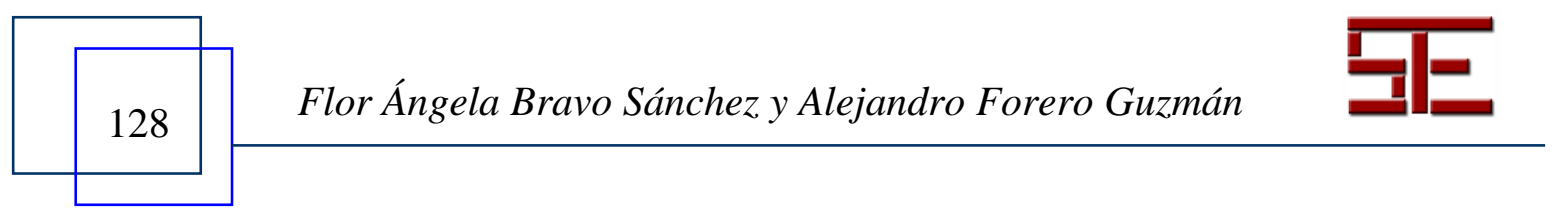




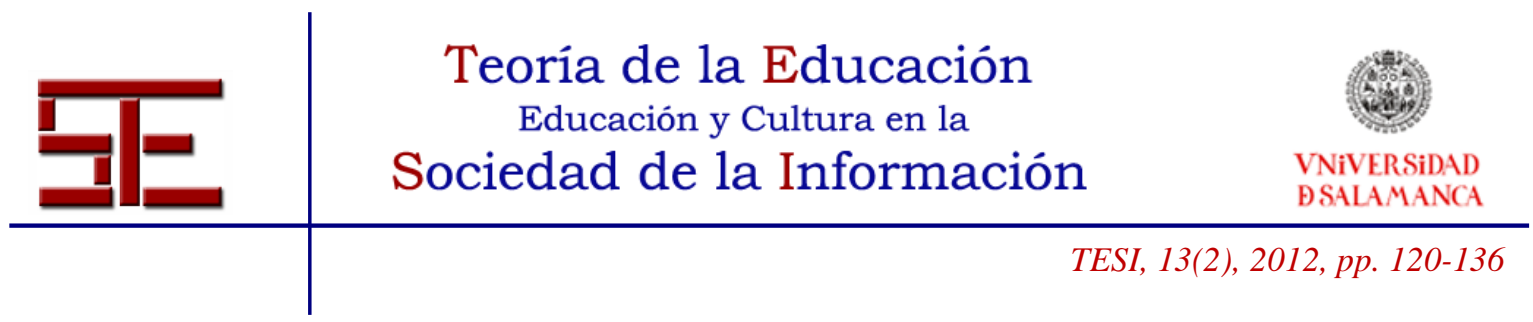

antes de ocuparse de la adquisición de las herramientas propiamente dichas; ese es el último paso dentro de esta etapa.

\section{4.- Etapa de definición del uso pedagógico de los recursos tecnológicos}

Contar con herramientas de aprendizaje basadas es robótica no es suficiente para la generación de ambientes de aprendizaje, hay que saber también cómo aplicar estos recursos adecuadamente en el aula de clase. El uso de estas herramientas debe estar acompañado de buenas prácticas pedagógicas para que puedan contribuir en los procesos de aprendizaje y la construcción del conocimiento de los estudiantes.

El diseño de actividades prácticas basadas en recursos robóticos permite definir un planteamiento pedagógico previo que guíe y regule el uso de estas herramientas. El desarrollo de estas actividades prácticas incentiva a los estudiantes a participar en la clase, generando interesantes ambientes de aprendizaje en donde pueden poner en práctica todos los temas vistos, crear sus

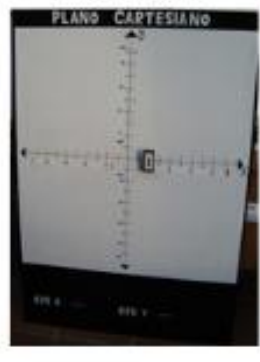

matemáticas basada en robótica.

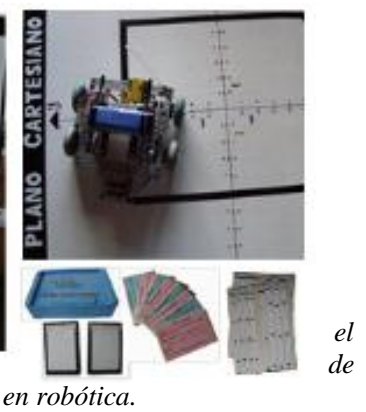
propias ideas de los conceptos que están siendo aplicados y al mismo tiempo relacionarlos con la realidad. El uso de un prototipo robótico en el desarrollo de las actividades fomenta en los estudiantes curiosidad, interés y concentración por los temas de clase.

Muchas veces es difícil para los docentes el diseño de estas actividades prácticas. Generalmente no tienen claro cómo usar estas herramientas en las asignaturas que dictan o no tienen el conocimiento del manejo de estas herramientas robóticas; y cuando estos inconvenientes están solucionados, el tiempo que le demanda a los docentes dar este cambio suele ser otro impedimento. Por esta razón es importante que personas expertas en el tema apoyen el diseño de actividades prácticas y la definición de los recursos de aprendizaje con todas las pautas necesarias para que puedan ser fácilmente replicadas por los docentes en el aula de clase.

Esta sería la última etapa en la implementación de un currículo que emplea cotidianamente la robótica educativa como una herramienta que simultáneamente es

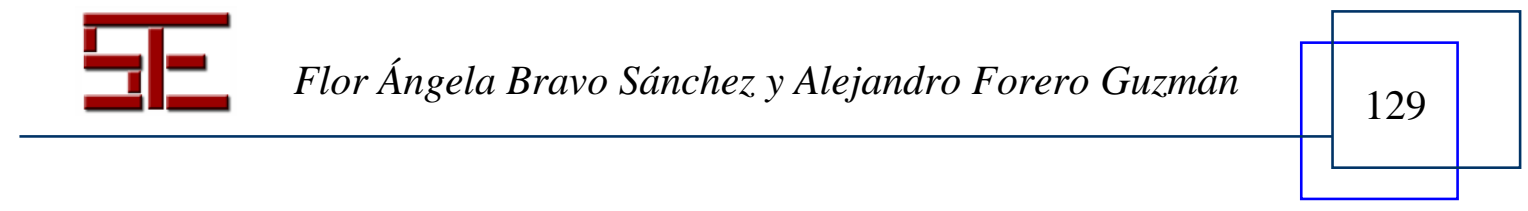




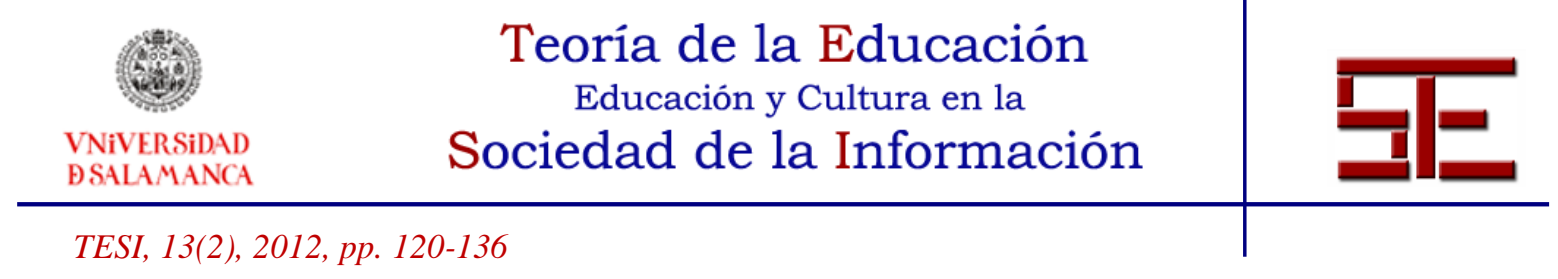

integradora, motivadora y a la vez el instrumento mismo con el cual se desarrolla la actividad en el aula, y posiblemente fuera de ella. Esta es una etapa que hasta ahora empieza a construirse impulsada por la suma de esfuerzos colectivos que diferentes instituciones empiezan a apoyar, un ejemplo de este confluir de actores se muestra en la sección siguiente.

\section{5.- CASO DE ESTUDIO: PROYECTO MUNDO ROBÓTICA}

La empresa de telecomunicaciones Telefónica S.A canaliza su Acción Social y Cultural través de la Fundación Telefónica. La estrategia de actuación de Fundación Telefónica se desarrolla a través de

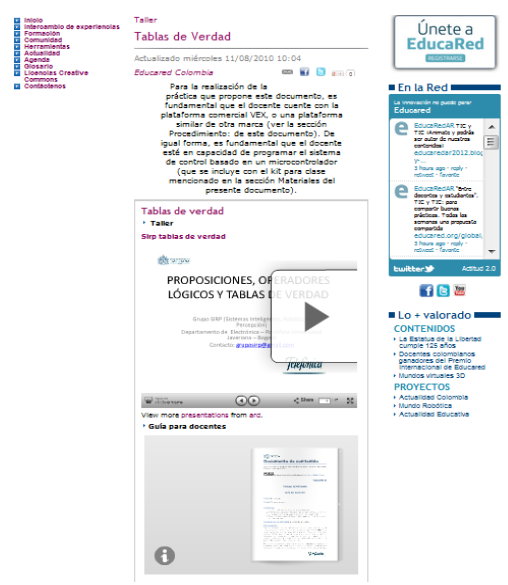

En la imagen se observa la sección Formación del portal MundoRobótica. diferentes programas cuyo objetivo es favorecer la implantación de las Tecnologías de la Información y la Comunicación (TIC) en los procesos educativos, potenciando la Sociedad de la Información del presente y del futuro en los diferentes países en donde el Grupo Telefónica está presente. Con dos ejes de trabajo, erradicación del trabajo infantil y promoción de la calidad de la educación, Fundación Telefónica articula sus esfuerzos en los siguientes programas: Proniño, Educared, Voluntarios Telefónica y Debate y Conocimiento (Ibarra; Arteaga; Maya, 2008).

Educared es un programa que Fundación Telefónica desarrolla en Argentina, Brasil, Chile, Colombia, Venezuela, España, México y Perú, para mejorar la calidad de la educación y fomentar la igualdad de oportunidades, mediante el uso de las TIC en los procesos de enseñanza y aprendizaje (Telefónica Colombia, 2008; EducaRed, 2009). Educared ofrece a los docentes, padres, estudiantes y demás personas relacionadas con la comunidad educativa capacitaciones y talleres sobre el uso de las TIC en la educación. También esta iniciativa cuenta con un portal educativo global con una gran variedad de contenidos y herramientas educativas.

El portal educativo EducaRed es un espacio para la generación de conocimiento, interactividad e interacción entre participantes de diferentes realidades y contextos.

Una de las iniciativas que tiene el portal educativo EducaRed para el fomento de las TIC en la educación es el proyecto Mundo Robótica. A continuación se detalla en qué consiste este proyecto.

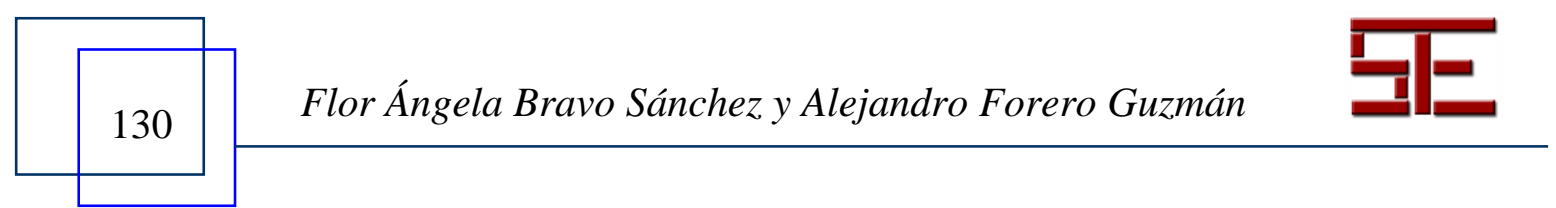




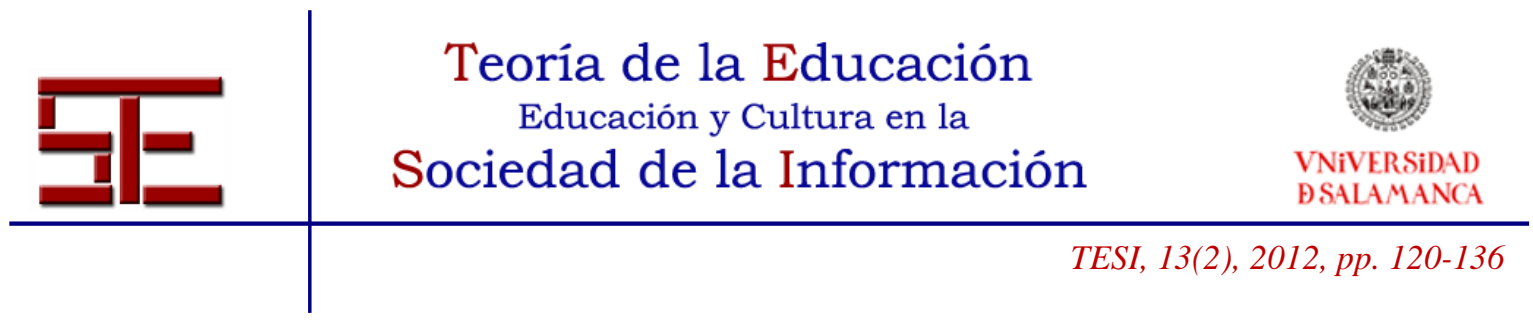

\section{1.- Mundo Robótica}

Mundo Robótica es una comunidad virtual de EducaRed (www.educared.org/global/mundo-robotica), especializada en la aplicación de la robótica en la educación. Se creó en 2008, en alianza con el Departamento de Electrónica de la Facultad de Ingeniería de la Pontificia Universidad Javeriana-Bogotá, con el objetivo de fomentar el uso de la robótica en el aula de clase como una herramienta de aprendizaje.

Mundo Robótica invita a la comunidad educativa y académica interesada en la temática a participar en un espacio virtual en el que pueden compartir sus experiencias, conocer nuevas prácticas de otros miembros de la red y construir conocimientos en torno a la robótica educativa. Los usuarios pueden poner en práctica los contenidos difundidos en Mundo Robótica en el ámbito educativo, para fortalecer el proceso de enseñanzaaprendizaje del estudiante y despertar su interés hacia la ciencia y la tecnología (Ibarra; Arteaga; Maya, 2008).

Los contenidos publicados en el portal de Mundo Robótica se distribuyen en siete secciones. A continuación se detalla cada una de ellas:

$\checkmark$ Intercambio de experiencias: Aquí los usuarios del portal de Mundo Robótica comparten sus experiencias de robótica educativa.

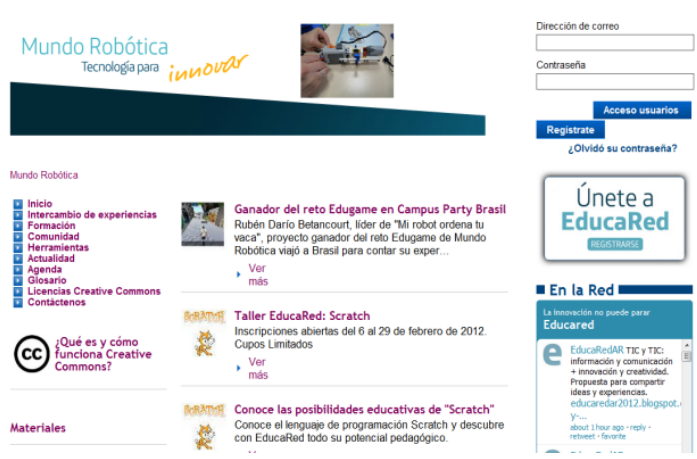

En la imagen se observa la página principal del Portal de Mundo Robótica (www.educared.org/global/mundorobotica). $\checkmark \quad$ Formación: El objetivo de esta
sección es proponer a los docentes de diferentes áreas actividades prácticas que involucren el uso de la tecnología, especialmente la robótica.

Un equipo de trabajo conformado por integrantes del grupo de investigación en Sistemas Inteligentes, Robótica y Percepción (SIRP) y estudiantes de pregrado y maestría en ingeniería electrónica de la Universidad Javeriana dedican sus esfuerzos al desarrollo de actividades prácticas y recursos de aprendizaje usando robótica que

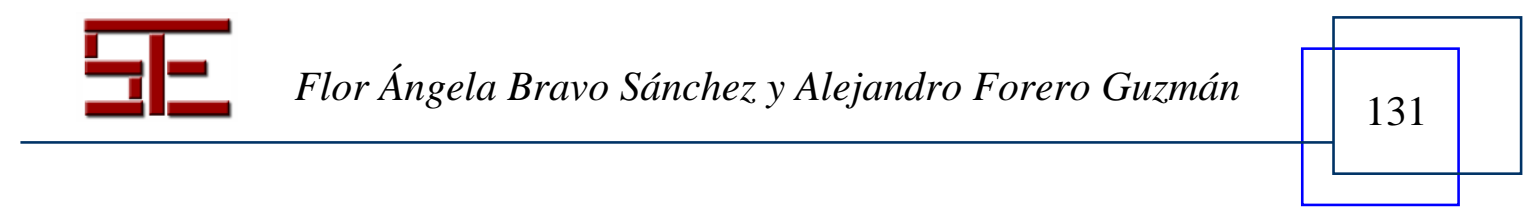




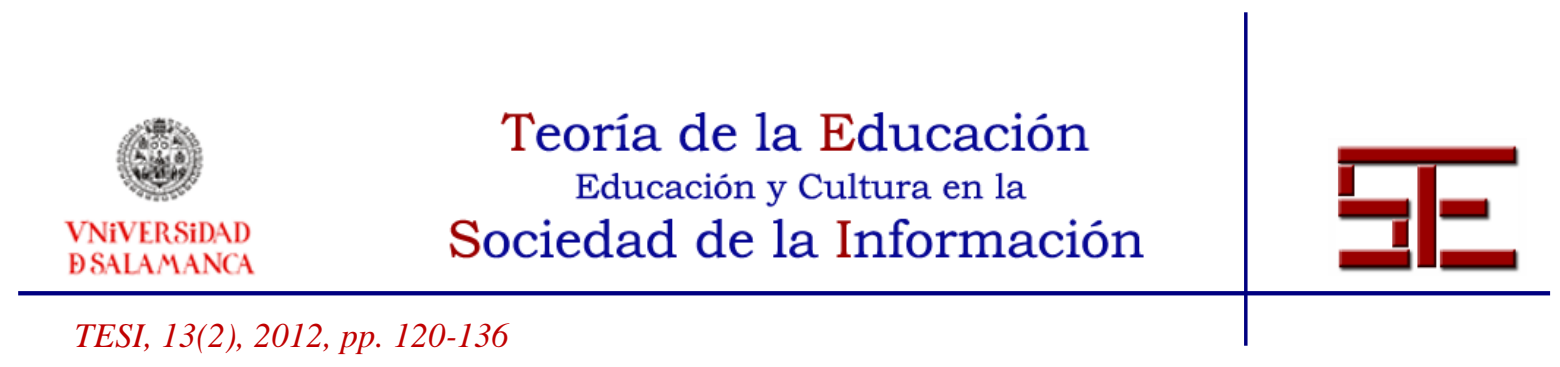

permitan al docente introducir sus temas de clase de una manera diferente y divertida para el estudiante.

En esta sección los usuarios encontrarán un listado de actividades prácticas basadas en robótica. Cada taller cuenta con un marco teórico, una guía para el estudiante y una guía para el docente con las pautas necesarias para el desarrollo del taller.

$\checkmark$ Comunidad: Esta sección está compuesta por un Blog y un foro. El Blog es administrado por el equipo de trabajo de la Javeriana (http://solorobotica.blogspot.com/).

$\checkmark$ Herramientas: Aquí el usuario encontrará un listado de kits recomendados para realizar los talleres propuestos en la sección de formación y algunas herramientas de software interesantes que pueden ayudar a la enseñanza de los temas de las diferentes asignaturas en el aula. El usuario también encontrará un listado de sitios web útiles en el aprendizaje y profundización sobre el mundo de la robótica y los lugares donde puede adquirir los materiales y componentes electrónicos para la construcción de prototipos robóticos.

$\checkmark$ Actualidad: En esta sección se publican las entrevistas realizadas a personalidades importantes del mundo de la robótica y artículos relacionados con la robótica educativa.

$\checkmark$ Agenda: Aquí se pueden consultar algunos eventos de robótica que se realizan a nivel nacional e internacional.

$\checkmark$ Glosario: En esta sección el usuario encontrará la terminología técnica empleada en los talleres, guías y obras publicadas en el portal.

Para incentivar el uso del portal de Mundo Robótica y la generación de una comunidad virtual en torno a la robótica educativa, se han desarrollado, desde los inicios del proyecto, una variedad de actividades presenciales a docentes y personas interesadas en la aplicación robótica en la educación. Con el apoyo del equipo de trabajo de la Javeriana se dictan talleres y conferencias sobre el uso de la robótica como una herramienta de aprendizaje. Estas actividades presenciales permiten a los docentes comprender las múltiples ventajas que trae el uso de estas herramientas en el proceso de aprendizaje del estudiante y lo fácil que es implementar este tipo de actividades en el aula de clase.

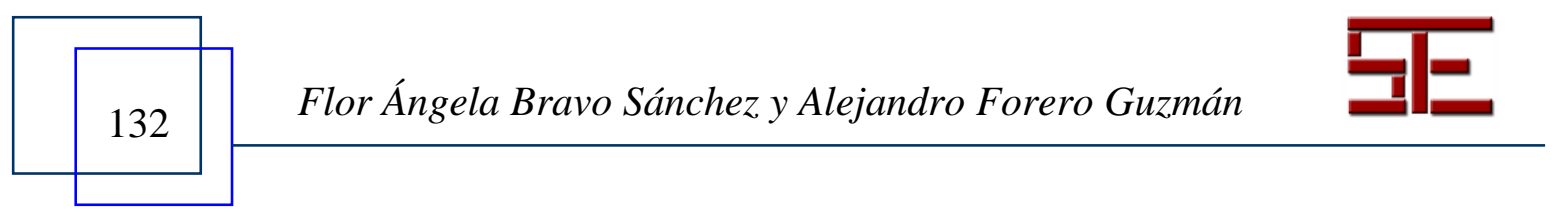




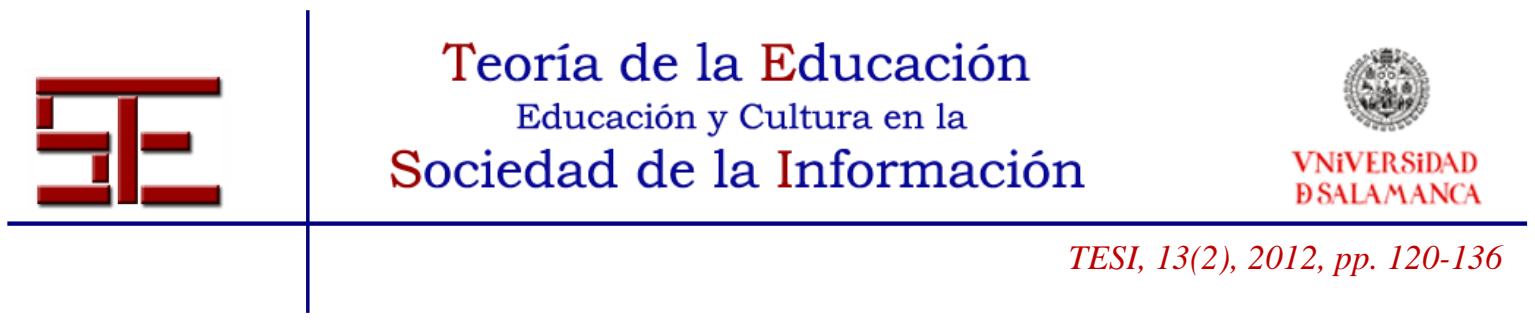

Durante el evento de Campus Party 2009 y 2010 se realizaron conferencias y talleres al público en general y en particular a grupos de profesores de todo el país. En estos talleres los profesores, asumiendo el rol de estudiantes, interactuaban con las herramientas de robótica y las usaban para aproximarse a diferentes áreas: aprendían un poco de historia, matemática o programación.

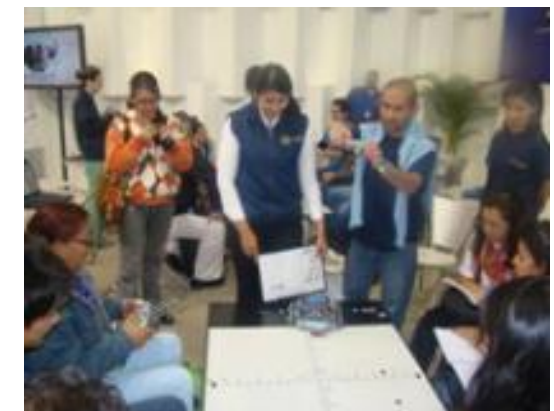

En la imagen se observa el desarrollo de uno de los talleres de robótica con profesores dictados en Campus Party Colombia 2009.

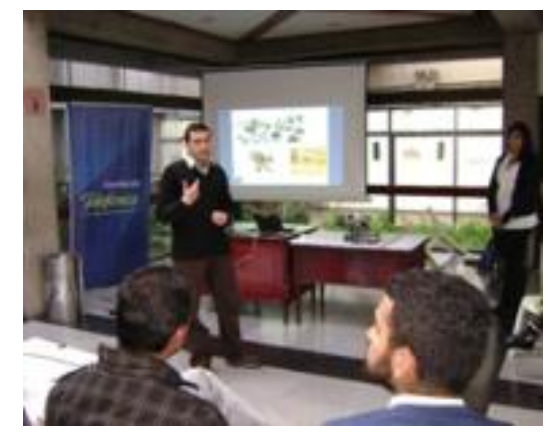

En la imagen se observa una de las conferencias de robótica educativa dictadas en Campus Party Colombia 2010.

\section{6.- CONCLUSIONES}

La sociedad actual está exigiendo al sistema educativo el desarrollo de nuevas habilidades y competencias que permitan a los estudiantes dar una respuesta eficiente a los entornos cambiantes del mundo actual. El uso de la robótica en el aula de clase como una herramienta de aprendizaje genera ambientes de aprendizaje multidisciplinarios que permiten a los estudiantes fortalecer su proceso de aprendizaje al tiempo que desarrollan diferentes destrezas que les permitirán afrontar los retos de la sociedad actual.

La robótica educativa tiene por objeto la concepción, creación y puesta en funcionamiento de prototipos robóticos y programas especializados con fines pedagógicos. La implementación de proyectos de robótica educativa en el aula de clase crea las mejores condiciones de apropiación de conocimiento, las cuales permiten a los estudiantes fabricar sus propias representaciones de los fenómenos del mundo que los rodea, facilitando la adquisición de conocimientos acerca de estos fenómenos y su transferencia a diferentes áreas del conocimiento.

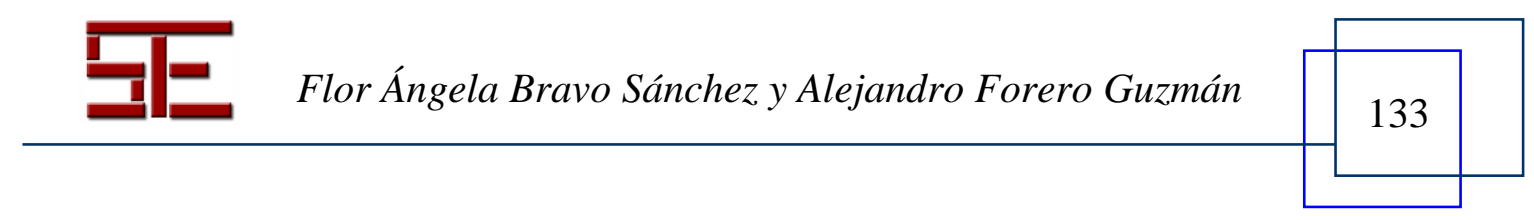




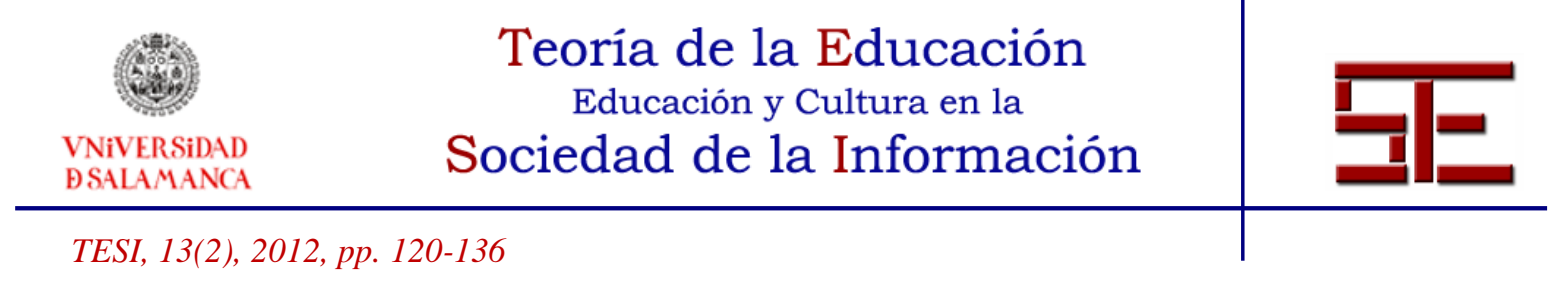

El proceso necesario para la implementación de proyectos de robótica educativa en el aula de clase puede dividirse en cuatro etapas. La etapa de integración de recursos tecnológicos basados en robótica al currículo busca superar el paradigma que se tiene de la robótica como una actividad extracurricular y reconocer la robótica como una herramienta de aprendizaje. Esta primera etapa plantea evaluar el plan curricular e involucrar proyectos basados en robótica en las diferentes asignaturas y fortalecer así el proceso enseñanza-aprendizaje del estudiante. La etapa de restructuración en las prácticas pedagógicas plantea establecer una nueva metodología de aprendizaje que fortalezca el proceso de enseñanza-aprendizaje del estudiante a través del uso de prototipos robóticos y programas especializados con fines pedagógicos. La etapa de instrumentación consiste en la adquisición de diferentes herramientas de software y hardware que permitan la construcción y programación de diferentes prototipos robóticos. Y, finalmente, la etapa de definición del uso pedagógico de los recursos tecnológicos plantea que este tipo de herramientas deben estar acompañadas de buenas prácticas pedagógicas para que puedan contribuir en la construcción del conocimiento de los estudiantes. Esta última etapa propone el diseño de actividades prácticas basadas en recursos robóticos, con el fin de tener un planteamiento pedagógico previo que guíe y regule el uso de estos recursos.

\section{7.- BIBLIOGRAFÍA}

Acuña, A. (2004). Robótica y aprendizaje por diseño. Extraído el 12 de febrero de 2012, de http://www.educoas.org/portal/ineam/premio/es58_2004.pdf.

- (2006). Projects for educational robotics: engines for the innovation. Current Developments in Technology-Assisted Education pp.951-956. Extraído el 6 de diciembre de 2011, de

http://www.fod.ac.cr/pdf/publicaciones/articulos/2006/951-956.pdf.

Aliane, N.; Bemposta, S.; Fernández, J.; Egido V. (2007). Una experiencia práctica de aprendizaje basado en proyecto en una asignatura de robótica. Extraído el 10 de diciembre de 2011, de http://bioinfo.uib.es/ joemiro/aenui/procJenui/Jen2007/ alunae.pdf.

Arduino (2006). Página de Internet. Extraído el 16 de enero de 2012, de http://www.arduino.cc/.

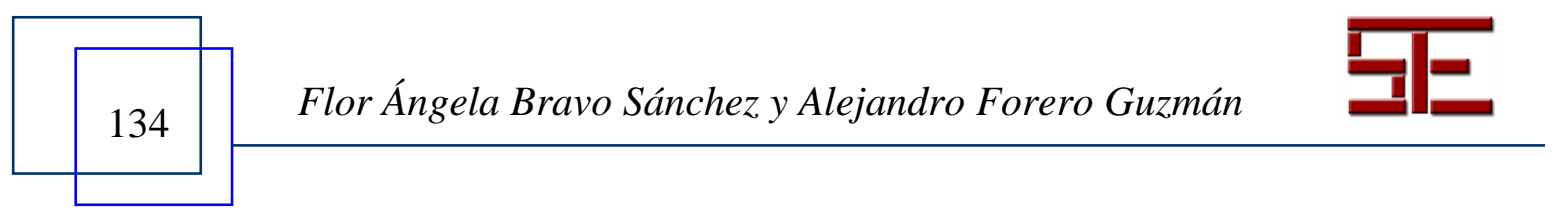




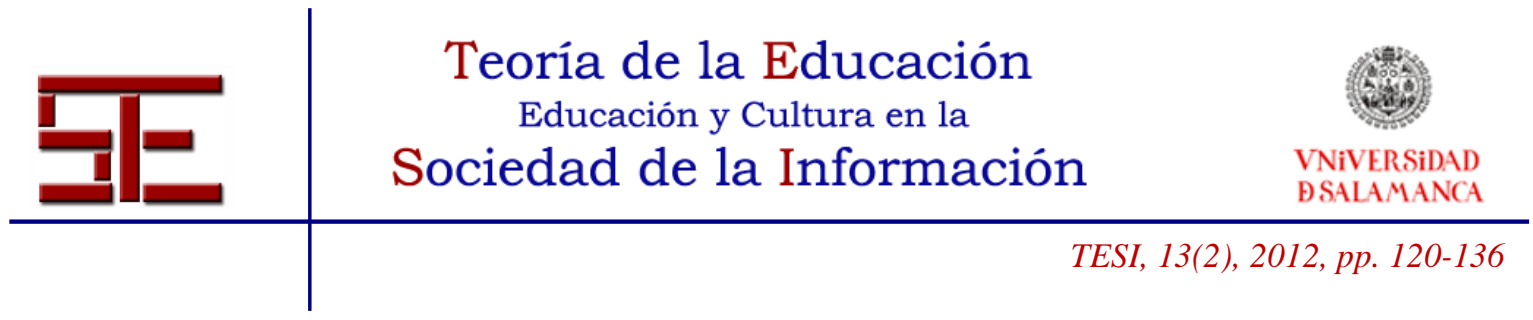

Computadores para Educar: Plataforma de Robótica y Automática Educativa (2008). Página de Internet. Extraído el 8 de abril de 2012, de http://www.computadoresparaeducar.gov.co/website/es/index.php?option=com_content $\&$ task=view $\&$ id $=173 \&$ Itemid $=285$.

Del Mar, A. (2006). Planificación de actividades didácticas para la enseñanza y aprendizaje de la ciencia y tecnología a través de la Robótica Pedagógica con enfoque CTS. Universidad Católica Andrés Bello, Caracas. Extraído el 5 de diciembre de 2011, de http://biblioteca2.ucab.edu.ve/anexos/biblioteca/marc/texto/AAQ6345.pdf.

EducaRed (2009). Página de Internet. Extraído el 15 de abril de 2012, de http://www.telefonica.co/fundacion/index.php?option=com_content\&task=view\&id=5.

Heli: Reciclaje y proyectos electrónicos (2012). Página de Internet. Extraído el 8 de abril de 2012, de http://heli.xbot.es/wp/?cat=3.

Hernández, R. (2008). El modelo constructivista con las nuevas tecnologías: aplicado en el proceso de aprendizaje. Revista de Universidad y Sociedad del Conocimiento (RUSC), vol. 5, n. ${ }^{\circ}$ 2. Extraído el 12 de febrero de 2012, de http://www.uoc.edu/rusc/5/2/dt/esp/hernandez.pdf

Ibarra, R.; Arteaga, G.; Maya P. (2008). Un ambiente de aprendizaje con la robótica pedagógica para embalaje. Extraído el 16 de enero de 2012, de: http://148.204.73.101:8008/jspui/bitstream/123456789/388/1/1_-_1-

IbarraQuevedoRaul.pdf.

Lrobotikas: Programación NXT (2011). Página de Internet. Extraído el 8 de abril de 2012, de http://lrobotikas.net/wiki/index.php?title=Programaci\%C3\%B3n_NXT.

Odorico, A. (2004). Marco teórico para una robótica pedagógica. Revista de Informática Educativa y Medios Audiovisuales, vol. 1(3), 34-46. Extraído el 12 de febrero de 2012, de http://laboratorios.fi.uba.ar/lie/Revista/Articulos/010103/ A4oct2004.pdf.

OPENQBO: Distribución Linux para Robots basada en Ubuntu (2010). Página de Internet. Extraído el 8 de abril de 2012, de http://thecorpora.com/blog/?p=335\&lang=es.

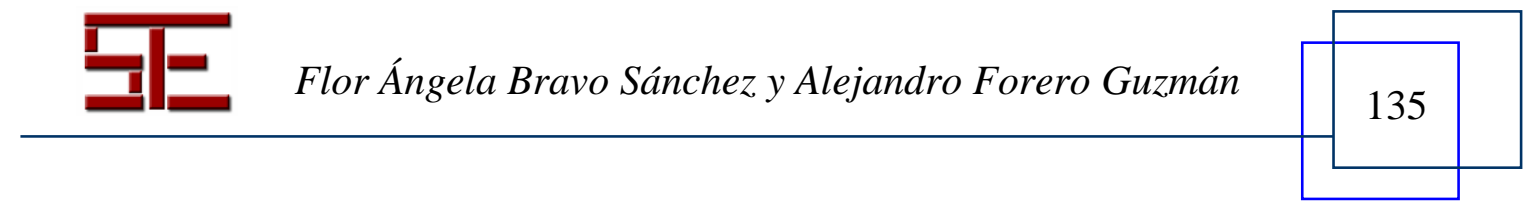




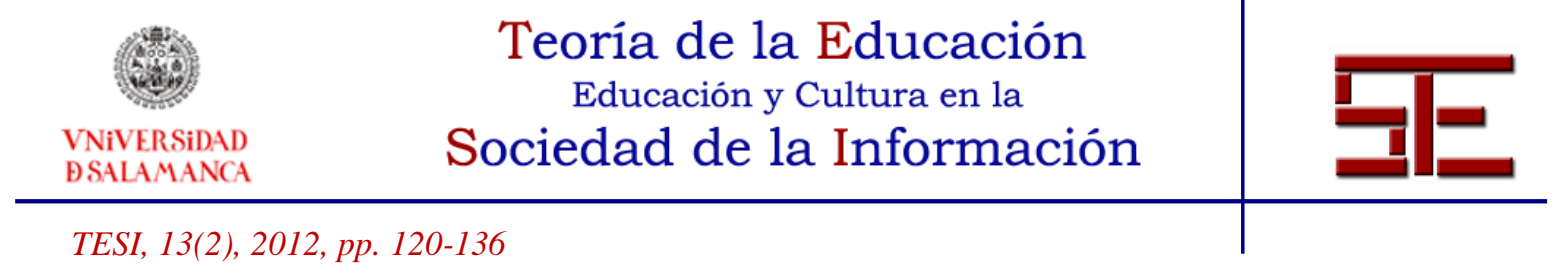

Ruiz, J. (2012). S4A Scratch + Arduino. Utilización de S4A (Scratch) más la tarjeta Arduino en un ambiente de programación gráfica orientado a la educación. Extraído el 6 de abril de 2012, de http://www.aprenderobotica.com/group/tutoriales/page/arduino.

Ruiz-Velasco, E. (2007). Educatrónica. Innovación en el aprendizaje de las ciencias y la tecnología. Madrid, España: Ediciones Díaz de Santos S.A.

Telefónica Colombia (2008). Informe Anual de Responsabilidad Corporativa Telefónica Colombia 2008. Extraído el 15 de abril de 2012, de http://www.telefonica.co/responsabilidadcorporativa/rc08/fp/cms/upload/ejecutivo_200 8_colombia.pdf.

Para citar el presente artículo puede utilizar la siguiente referencia:

Bravo Sánchez, F. A. y Forero Guzmán, A. (2012). La robótica como un recurso para facilitar el aprendizaje y desarrollo de competencias generales. Revista Teoría de la Educación: Educación y Cultura en la Sociedad de la Información. 13(2), 120-136 [Fecha de consulta: $\mathrm{dd} / \mathrm{mm} / \mathrm{aaaa}$ ].

http://campus.usal.es/ revistas_trabajo/index.php/revistatesi/article/view/9002/9247

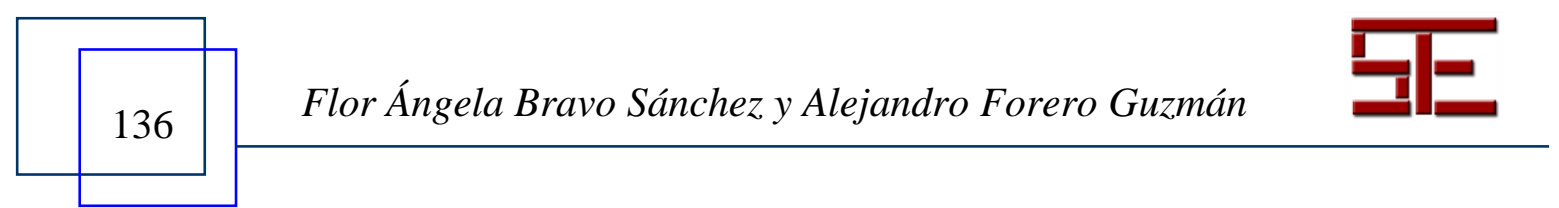

